\title{
Success Rate of Salvage Surgical Intervention of Acutely Thrombosed Arteriovenous Fistula in Hemodialysis Patients
}

\author{
Mohamed M. El Yamany*, Hatem H. Mohamed, Micheal N. El Abd, and \\ Sherif A. Reffat
}

Department of Surgery, Vascular and Endovascular Unit, Suez Canal University Hospital, Ismailia, Egypt

\begin{abstract}
Background: Establishing and maintaining vascular access for hemodialysis in end stage renal disease (ESRD) patients are important prognostic factors for patient survival. The loss of vascular access, most frequently due to thrombosis, remains one of the most challenging obstacles in their long-term management. Success rate of surgical intervention of the vascular access in patients with acutely thrombosed arteriovenous fistulas (AVF) is still a controversial issue. Objective: To assess the success rate of salvage of acutely thrombosed arteriovenous fistula through surgical intervention. Patient and Methods: Uncontrolled clinical trial, was conducted over the period from November 2018 to 'October 2019 on 52 patients with acute thrombosis of autogenous AVF in Suez Canal University Hospital. Patients were treated using open thrombectomy with or without surgical interventions as jump graft or re-insertion of anastomosis to correct juxta-anastomosis stenosis or re-anastomosis if needed regarding the cause. Results: The mean age was $51+/-14$ years. The cause of thrombosis was stenosis in 13 patients (25\%). Surgical intervention was successful to regain immediate functioning AVF in 34 patients (65.4\%). The secondary patency rate after intervention was more than 6 months in 29 patients (85.3\%). Jump graft with surgical thrombectomy in treatment of stenosis as a cause of thrombosis was the highest rate success in 8 patients (88.9\%). Conclusion: Intervention with different surgical modalities for acutely thrombosed AVFs is a safe and effective procedure in many cases. It is associated with good success rates, low complication rates, and maintained long-term patency of vascular access.
\end{abstract}

Key words: Arteriovenous Fistulas, Hemodialysis, Thrombectomy

\section{Introduction}

The hemodialysis vascular access may be autogenous arteriovenous fistulas (AVF) or prosthetic arteriovenous grafts (AVG) or central venous catheters placed in a large vein. The autogenous AVF is usually created by joining a superficial vein to an adjacent peripheral artery at the level of the wrist or in the mid forearm; AVFs are the preferred initial hemodialysis access due to their longer patency than AVGs(1). Maintaining a patent vascular access remains a major challenge, especially in an aging hemodialysis

*Corresponding Author: mohamed_alyamany@med.suez.edu.eg 
population. Thrombosis of the hemodialysis access is the most frequent complication encountered in vascular access surgery that can lead to loss of the vascular access. The most common sites of thrombosis are juxtaarteriovenous anastomosis or along the proximal venous limb ${ }^{(1)}$. Dealing with hemodialysis access complications and restoring its normal function could be done either with open surgery or endovascular procedures. The most efficacious technique for access salvage is still unknown ${ }^{(2)}$. So, we design this study to assess the success rate of salvage surgical intervention of acutely thrombosed arteriovenous fistula.

\section{Patients and Methods}

This is uncontrolled clinical trial in Suez Canal University Hospital over a period from November 2018 to the end of October 2019. 52 Patients with ESRD that developed vascular access failure due to acute thrombosis or pre-dialysis patients with thrombosed vascular access in Suez Canal University Hospital. All patients presented with infected AVF, aneurismal dilatation in the vascular access and central venous pathology were excluded from the study. Inclusion criteria: i) A totally occluded autogenous access without thrill or bruit as seen by physical examination. ii) Confirmed thrombus by Duplex ultrasound examination. iii) Inability to be used for hemodialysis. Time of surgical thrombectomy operation is within 48 hours from access thrombosis time. iv) Patients with normal coagulation profile "PT, PTT, INR, platelet count". Exclusion criteria: i) Patients with decreased blood pressure (Systole <90). ii) Patients with infected AVF or graft. iii) Patients with thrombosed aneurysmal dilatation in the hemodialysis access. iv) Patients with central venous stenosis or occlusion. The project was ethically approved by the
Institutional Ethical Committee of the faculty of medicine, Suez Canal University. Informed consent was obtained from all individual participants included in this study. Any patient with thrombosed AVG, central venous stenosis or having coagulation disorders is excluded from this study.

Pre-operative assessments

- Medical history: included history of present illness, detailed medical history, risk factors, allergy, and type of AVF, history of previous vascular operations and the exact time of lost thrill.

- Physical Examination: included Blood pressure, chest and heart examinations AVF examination: presence of palpable and /or audible thrill, soft or hard thrombus, the length of thrombosed segment, distance from anastomosis, the presence of dilated segments and signs of infection or hematoma.

- Investigations: included CBC, PT, PTT, INR, random blood sugar, $A B G$, serum creatinine, sodium, and potassium. ECG, and Duplex for confirmation of diagnosis, echogenicity, length of thrombosed segment and causes (anastomosis size, central venous occlusion, and stenosis).

\section{Procedure}

The procedure was performed under local anesthesia over the thrombosed segment. A skin and subcutaneous incision $(2-3 \mathrm{~cm})$ to access the vein was made proximal and distal to the control of the vein. A small oblique incision was made in the vein juxtaanastmosis or at the venous. A 3 Fr. Fogarty catheter was used to perform thrombectomy from the oblique incision, in both ante-grade and retrograde directions. After thrombectomy, excellent arterial inflow was seen. Closure of the venotomy using simple sutures of polyproline 6/0 was done. 
Hemostasis and skin closure.

If there was doubt in the anastomosis, revision of the anastomosis was done to make sure that it was not anastomotic problem. If tight stenosis juxta-anastmosis was found and the rest of the vein was patent proximal reinsertion of the vein to the artery was done. Patient was discharged the following day. Follow up was done after 3, 10 days, 1, 3 and 6 months, checking for: thrill, suitability for hemodialysis (which would start for the rest of the vein after 4 days), infection, hematoma and re-thrombosis.

\section{Statistical analysis}

The results were analyzed using SPSS version 20. Non-parametric tests were used for analysis; this group differences were assessed using Kruskal-Wallis and Mann-Whitney $\mathrm{U}$ test. Likert score averages were given as Mean $\pm \mathrm{SD}$. $\mathrm{P} \leq 0.05$ was set as a criterion for establishing statistical significance.

\section{Results}

Between November 2018 to October 2019, 52 patients with acutely thrombosed autogenous arteriovenous fistulae presented to the vascular surgery unit in Suez Canal University Hospital. They underwent open thrombectomy procedures, 34 (65.4\%) involved an upper arm AV fistula and 18 (34.6\%) fore-arm RC AV fistula. Among the previously mentioned $(n=34,65.4 \%)$ cases of upper arm AVF, they included brachio-basilic AVF $(n=8,15.4 \%)$ and brachio-cephalic AVF $(n=26,50 \%)$ as well. In table 1 , the demographic data were described including the patient's chronic illness. The pre-intervention (primary patency) of all vascular accesses our study was described in figure 1 . In table 2, the causes of thrombosis and number of patients regarding each cause were mentioned. Many of surgical intervention have been used to fix the cause of thrombosis as thrombectomy alone or with reanastomosis or bypass using jump graft or reinsertion of the anastomosis as described in Figure 2. The success rate of our intervention was described in Figure 3. After intervention we follow-up the patients for 6 months and the secondary patency rate was evaluated in figure 4 regarding the duration. And at the end of the results figure 5 describes the success rate of each technique post intervention.

\section{Intervention}

A 3 Fr. Fogarty catheter was used to perform thrombectomy from the oblique incision, in both ante-grade and retro-grade directions in all patients. In 29 patients (55.8\%) only thrombectomy by a 3 French Fogarty catheter was done, we had done re-anastomosis in 6 patients (11.5\%) of early thrombosis usually in the first day after fistulae creation. A 6 mm PTFE synthetic jump graft was inserted over a short segment that wasn't completely patent and flow limiting after thrombectomy to bypass it in 9 patients (17.3\%) of arm AVF (brachio-cephalic and brachio-basilic with vein transposition. In another 8 patients (15.4\%) with Radio-cephalic AVF who had thrombectomy and got patent vein but tight anastomotic stenosis we did proximal reinsertion of the vein to the radial artery.

\section{Discussion}

The burden of ESRD is growing dramatically over the recent few years not only on the national level in Egypt but also worldwide(3). As a chronic disease and involving young age groups; recent studies are made to treat the complications of the limited hemodialysis accesses available for each patient aiming to increase their life expectancy ${ }^{(4)}$. 
Table 1: Demographic data of the participants

\begin{tabular}{|l|c|}
\hline Total No. of Patients (N=52) & No. $(\%)$ \\
\hline Age in years mean \pm SD & $51 \pm 14$ \\
\hline Male & $34(65.4 \%)$ \\
\hline Female & $18(34.6 \%)$ \\
\hline Diabetes & $22(42.3 \%)$ \\
\hline Hypertension & $26(50 \%)$ \\
\hline IHD & $12(23.1 \%)$ \\
\hline SLE & $6(11.5 \%)$ \\
\hline HCV +VE & $8(15.4 \%)$ \\
\hline
\end{tabular}

Keeping the vascular access patent and safe through the process of hemodialysis is the main hope of both surgeon and patient, thrombosis of this access will affect the life of these patients, so this was the aim of this study to find a solution for this complication(4). This study was conducted to assess success rate of different modalities of surgical intervention in the management and salvage of acutely thrombosed AVF aiming to extend the patency rate of AVF and to restore its function in hemodialysis. Our study showed that male to female ratio was 2:1. 36 patients of the were males (67.3\%) and 18 patients were females (33.7\%), on the other hand other studies such as Ghaffarian et al study $(5)$, had (77\%) of the study subjects were males and (23\%) were females with a ratio of 3:1 aligned with male predominance, which is in agreement with our results. Other studies such as Ponikvar et al study(6), showed that male to female ratio was nearly equal; as (47.3\%) of the candidates were men and (52.7\%) were women ${ }^{(6)}$. Regarding the risk factors for thrombosis; our study showed a significant correlation between cardiovascular diseases including ischemic heart disease and hypertension and thrombosis in hemodialysis access; (59\%) of patients included in our study who presented with non-functioning AVF had a history of cardiovascular
Table 2: The cause of thrombosis

\begin{tabular}{|l|c|c|}
\hline Cause of thrombosis & Frequency & Percent \\
\hline Compression & 8 & $15.4 \%$ \\
\hline Intimal hyperplasia & 10 & $19.2 \%$ \\
\hline Hypotension & 12 & $23.1 \%$ \\
\hline Stenosis & 13 & $25 \%$ \\
\hline Unknown & 9 & $17.3 \%$ \\
\hline Total & 52 & $100.0 \%$ \\
\hline
\end{tabular}

disease. This may be because hypertension increases the shear stress upon the constructed AVF, in addition to atherosclerosis enhancing the process of neo-intimal formation resulting in thrombosis. In our study we showed overall success rate (65.4\%) evidenced by post-operative palpable thrill and restoration of its function in hemodialysis, (9.6\%) of them got rethrombosis through the first 6 months of follow up, (55.8\%) patients kept patent functioning AVF for more than 6 months, which means that the chance of the patients who underwent to these trial of surgical interventions through different modalities had high possibility of keeping their shunts work safe and patent, Similar results reported by Franco et al study(7) 45 salvage procedures were performed in 41 accesses of 40 hemodialysis patients with native AVF. Of these, 90\% were AVF, mostly upper arm. Clinical success rate was $60 \%$. They studied primary patency for 12 months and it was $39 \%(7)$. Regarding the type of occlusion among those fore-mentioned successful cases; among the (55.8\%) of patients who kept patent functioning AVF for more than 6 months (18.5\%) of them had primary occluded AVF, while among the (9.6\%) of patients who got rethrombosis through the first 6 months of follow up, (3.5\%) of them had a primary occluded AVF. 


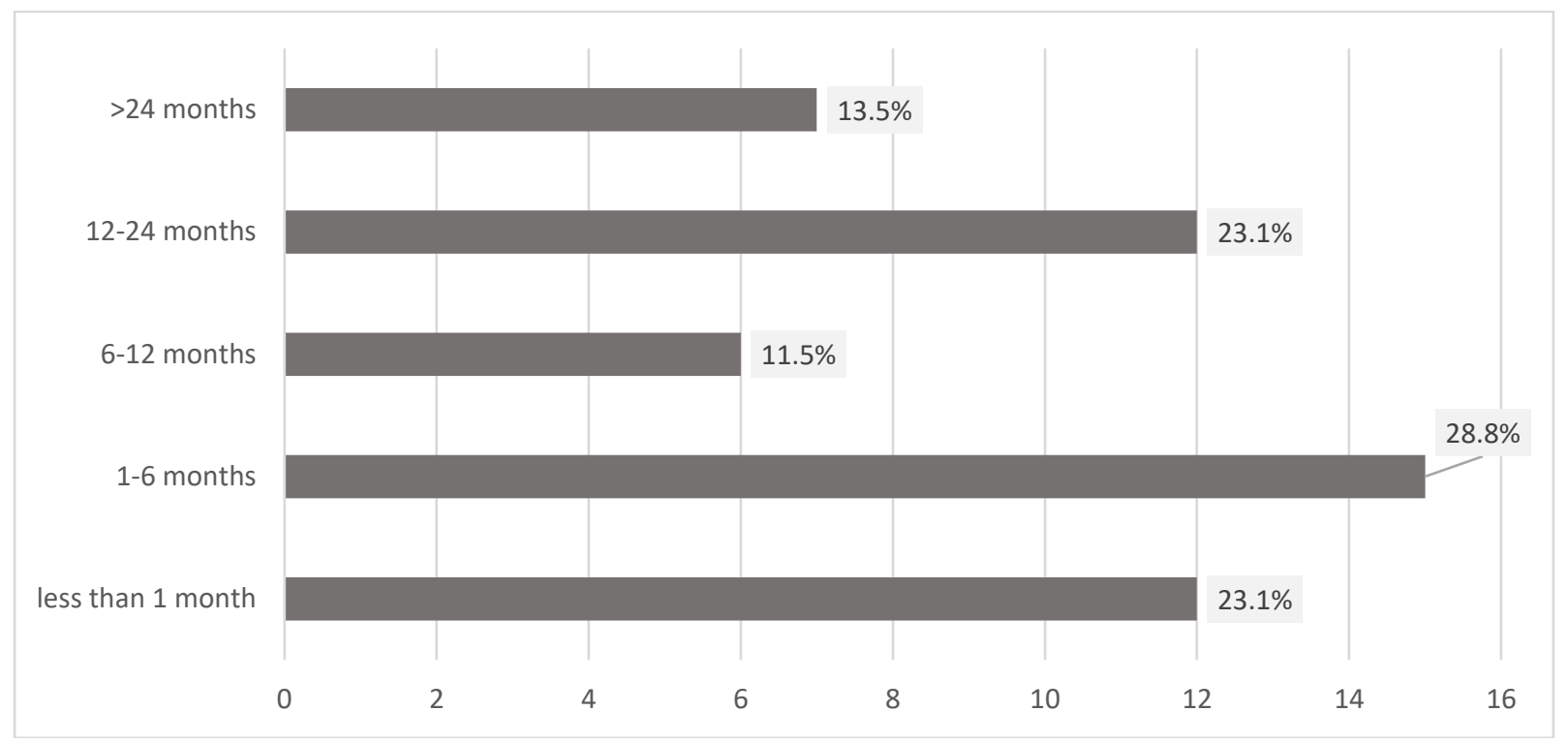

Figure 1: Pre-intervention (Primary patency) of AVF

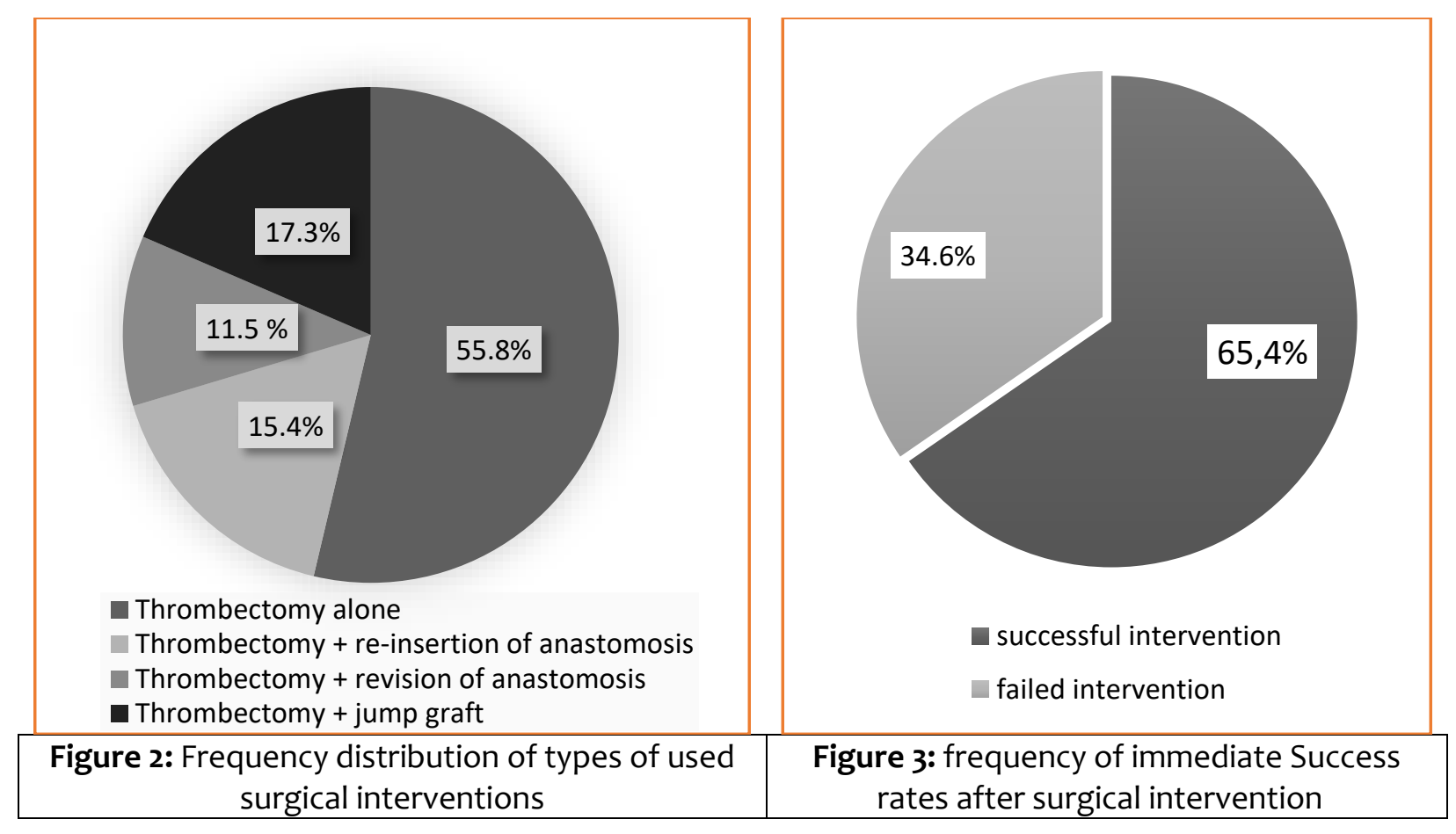

Similar results reported by Franco et al study ${ }^{(7)}, 45$ salvage procedures were performed in 41 accesses of 40 hemodialysis patients with native AVF. Clinical success rate was (60\%). They studied primary patency for 12 months and it was (39\%)(7). 


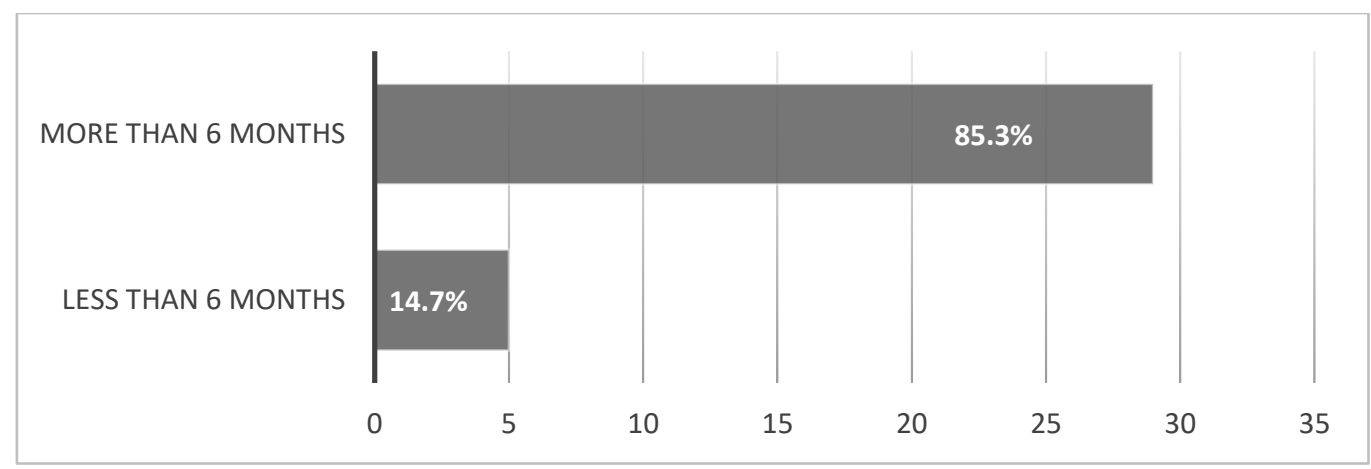

Figure 4: Secondary patency rates among successful cases

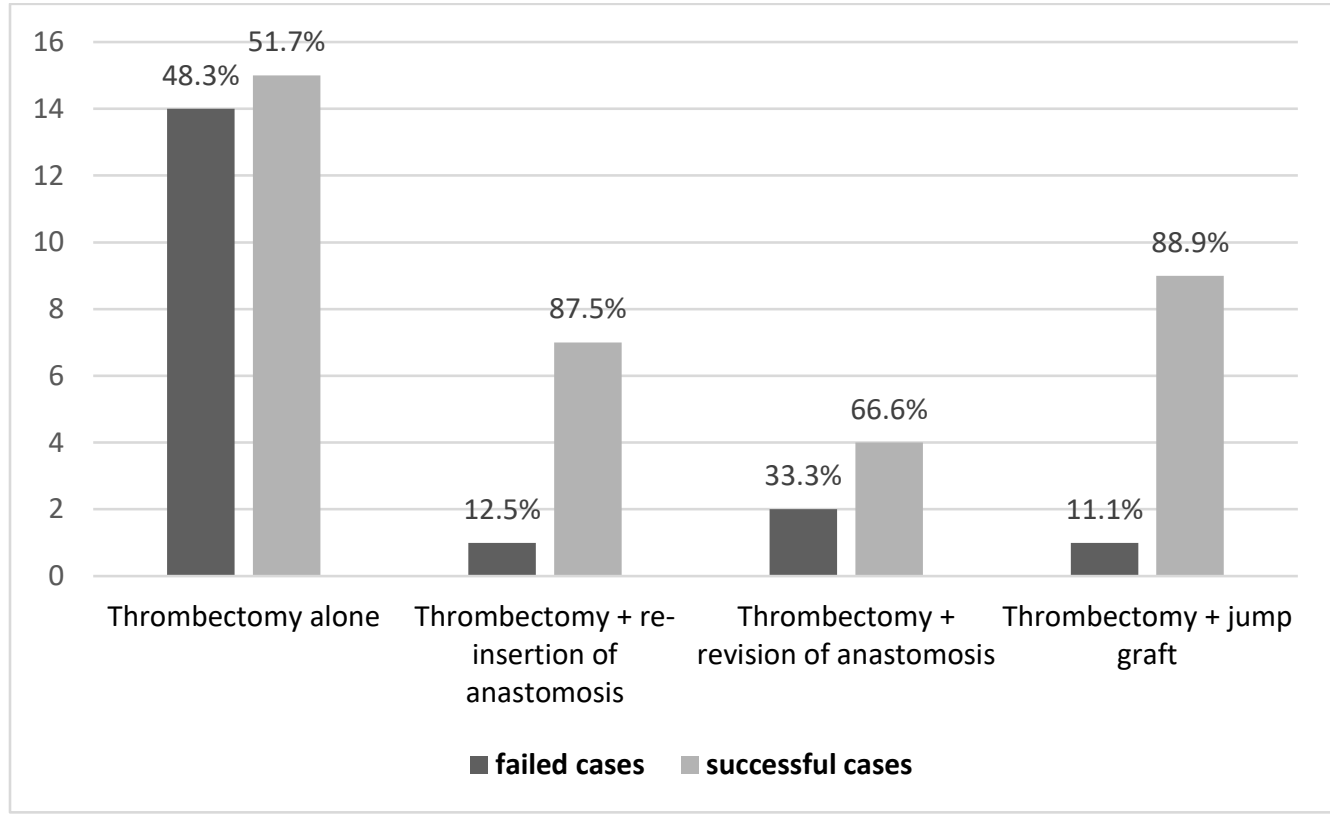

Figure 5: success rate of different surgical procedures

In our study 23 patients (44.2\%) the causes of acute thrombosis were stenosis (25\%) and hyperplasia (19.2\%),that needed additional procedure of intervention with thrombectomy to fix these defects, jump graft (PTFE 6mm) was used to bypass the stenotic segment in arm AVFs due to the sizable vein of that anatomical area while re-insertion of the site of anastomosis was the procedure added to thrombectomy to fix the juxta anastomotic stenosis in radio-cephalic AVFs while re-anastomosis was added to thrombectomy in the patients who have acute thrombosis of their AVFs in the first day as we suspect technical problem. This briefly explain that stenosis is mostly related to recurrent insertion of the needles of hemodialysis in the same sites which lead to endothelial injury and starts the cascade of intimal hyperplasia and wall thickness which also usually associated with atherosclerosis in the long period which will cause thrombosis later. Ghaffarian et al study ${ }^{(5)}$ showed that $80 \%$ of their candidates had venous 
outflow stenosis while $5 \%$ had stenosis at the arterial anastomosis and this indicates that stenosis is an important reason for thrombosis as it experiences inflow and outflow stenosis more commonly(5). We found that the success rate of the thrombectomy alone as the only surgical intervention was done to 29 patients was (51.7\%), these patients had no apparent cause of thrombosis through examination and through exploration as stenosis, intimal hyperplasia or anastomotic problem. while when we used additional procedures to the intervention of thrombectomy, the success rate was improved dramatically as when we used jump graft in arm fistulas to bypass the stenotic segment of the cephalic or basilic veins success rate was (88.9\%). When we used re-insertion procedure with thrombectomy in radio-cephalic AVFs success rate was (87.5\%) and lastly when we added re-anastomosis to thrombectomy in early thrombosed AVFs after new creation, the success rate was (66.6\%), this mean that there is a need to add another surgical intervention if the causes were stenosis, hyperplasia and early thrombosis after creation of AVFs since thrombectomy alone is not enough and its failure rate was high. Unfortunately, there is no available studies to compare these results with them. Patients in the current study with mature AVF were able to re-use their AVF for hemodialysis after 4 days' post-operative and the maturing AVF were left till maturation and were used afterwards. Post-operative complications reported such as anastomosis rupture, compressing neither hematoma nor anaphylactic shock. No case reported infection or active bleeding post-operative during the follow up period. Meanwhile, in Franco et al study reported similar results as there were not any post-operative major complications $^{(7)}$.

\section{Conclusions}

Intervention with different surgical modalities for acutely thrombosed AVFs is a safe and effective procedure. It is associated with high success rates, low complication rates, and maintained long-term patency of the hemodialysis vascular access.

Financial support and sponsorship: None

Conflict of interest: None

\section{References}

1. Tang S, Lo CY, Tso WK, Lo WK, Li FK, Chan TM. Percutaneous transluminal angioplasty for stenosis of arteriovenous fistulae: a review of local experience. Hong Kong Med J.1998;4(1):36-41.

2. Andziak P. Ultrasound-guided angio-plasty of dialysis fistula-technique description. Polish J Radiol. 2013; 78 (4):56-61.

3. Sidawy AN, Spergel LM, Besarab A, et al. The Society for Vascular Surgery: Clinical practice guidelines for the surgical placement and maintenance of arteriovenous hemodialysis access. J Vasc Surg. 2008;48(5 SUPPL.):2-25.

4. Faiyaz R, Abreo K, Zaman F, Pervez A, Zibari $G$, Work J. Salvage of poorly developed arteriovenous fistulae with percutaneous ligation of accessory veins. Am J Kidney Dis. 2012;39(4):824-827.

5. Ghaffarian AA, Sarfati MR, Kraiss LW, et al. Open thrombectomy of arteriovenous fistulas: Worth the effort or is it time to move on ? J Vasc Surg. 2017;66 (3):e45-6.

6.Ponikvar R, Premru V, Kersnic B. Surgical Thrombectomy of Thrombosed Arteriovenous Grafts by Interventional Nephrologists. Ther Apher Dial 2011;15 (3):306-10.

7. Franco RP, Chula DC, Alcantara MTD, Rebolho EC, Melani ARA, Riella MC. Salvage of thrombosed arteriovenous fistulae of patients on hemodialysis: report on the experience of a Brazilian center. Braz J Nephrol. 2018; 40(4), 351-359. 